\title{
Sciatica: Internet Search Trends
}

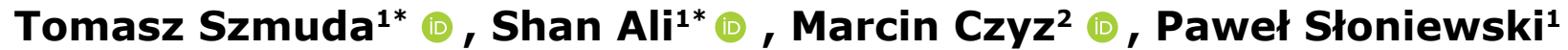

\author{
${ }^{1}$ Scientific Circle of Neurology and Neurosurgery, Neurosurgery Department, Medical University of Gdańsk, Poland \\ ${ }^{2}$ Royal Orthopaedic Hospital, Birmingham, United Kingdom \\ *contributed equally to the manuscript
}

\begin{abstract}
Background: Sciatica is a significant global health burden with a lifetime incidence estimated between $13 \%$ and $40 \%$. In our study, we assessed the online health interest in sciatica and lower back pain. Material and methods: Google Trends, Wikipedia statistics and PubMed data were used to gauge the online public interest in sciatica and lower back pain. Results: In the years 2015-2019 the Wikipedia page about sciatica has ranked high in all four categories it was included in, thus demonstrating that sciatica is a significant concern for the public. Wikipedia pages about sciatica and low back pain had a respectively $28 \%$ and $90 \%$ increase in views from July 2015 to March 2019. In the last eleven years (2008-2019) Google Trends demonstrates that sciatica has had a 2-fold increase in search frequency worldwide on the web, a 6-fold increase on YouTube and a 3-fold increase on Google images. In contrast, scientific interest in sciatica is low (only 140 PubMed publications in 2018). Conclusions: People have a relatively high and increasing online interest concerning sciatica and back pain. As a response, we suggest that hospital staff clearly provide reliable and understandable information to their patients concerning sciatica and lower back pain treatment.
\end{abstract}

Keywords: lower back pain · sciatica · Google Trends · Wikipedia

\section{Citation}

Szmuda T, Ali S, Czyz M, Słoniewski P. Sciatica: Internet Search Trends. Eur J Transl Clin Med. 2020;3(1):49-52.

DOI: $10.31373 /$ ejtcm/119130

\section{Introduction}

Majority of patients use online resources such as Wikipedia and a Google search to learn more about their illnesses. Wikipedia, a free online encyclopedia, is one of the leading sources of healthcare informa- tion [1-2]. Google is the world's most popular search engine which produces a significant portion of online searches for information about diseases. Both these platforms collect and publish statistics on user search traffic and this information may be used for analysis. Additionally, PubMed is an online database that

Corresponding author:

Shan Ali, Scientific Circle of Neurology and Neurosurgery, Neurosurgery Department, Medical University of Gdańsk, Poland

e-mail: shanali@gumed.edu.pl

No external funds.

Available online: www.ejtcm.gumed.edu.pl

Copyright $®$ Medical University of Gdańsk

This is Open Access article distributed under the terms of the Creative Commons Attribution-ShareAlike 4.0 International. 
indexes all major biomedical journals and it may be used to estimate the scientific output on a particular topic. So far, studies have been conducted using these platforms to assess the overall trends of several neurological diseases including epilepsy and multiple sclerosis [3-5].

However, these online tools have not yet been used to assess the online search trends for sciatica. Hippocrates first published about "sciatic pain" more than two thousand years ago, whereas in the early 20th century Harvey Cushing helped to establish surgical treatments for this condition. Today, sciatica is a significant global health burden with a lifetime incidence estimated between $13 \%$ and $40 \%$ [6]. Given its high incidence, our aim was to assess the popularity of sciatica online using Google Trends, Wikipedia and PubMed publication data [7-8].

\section{Methods and materials}

The Wikipedia Massviews tool was used to assess the objective popularity of sciatica in the following 4 categories it was indexed under: symptoms and signs: musculoskeletal system, orthopedic problems, pain, and peripheral nervous system disorders. Sciatica was searched (as a medical condition) on Google Trends for the last full eleven years (2008-2019). PubMed results were used to estimate the number of scientific articles on sciatica for the year 2018 the following keywords were searched: "sciatica," "low back pain" and "leg pain AND lumbar AND 2018."

\section{Results}

\section{Wikipedia findings}

Over the last full five years, (2015-2019) the Wikipedia article on sciatica has ranked high in all of the 4 categories it was included in: it ranked first under Symptoms and signs: musculoskeletal system, first under Orthopedic problems, first under Pain and second under Peripheral nervous system disorders. Considering that Wikipedia is the number one source for health information for patients and health care professionals, this demonstrates that sciatica is a significant concern for the public [1]. Sciatica not only seems to be more of a concern compared to other neurological disorders, like trigeminal neuralgia or neuropathic pain, but it is the most commonly searched topic in the vast category of "pain." Furthermore, Wikipedia page views on sciatica and low back pain show a $28 \%$ and $90 \%$ increase respectively from July 2015 to March 2019.

\section{Google findings}

In the last full eleven years (2008-2019) Google Trends shows that sciatica (as a medical condition) had a 2-fold increase in search frequency worldwide on the web, a 6-fold increase on YouTube and a 3-fold increase on Google images. Lower back pain (as a disorder) exhibited a similar trend with a 2 -fold increase in search frequency worldwide on the web, 8-fold increase on YouTube (see Figure 1) and a 3-fold increase on Google images from the same time period.

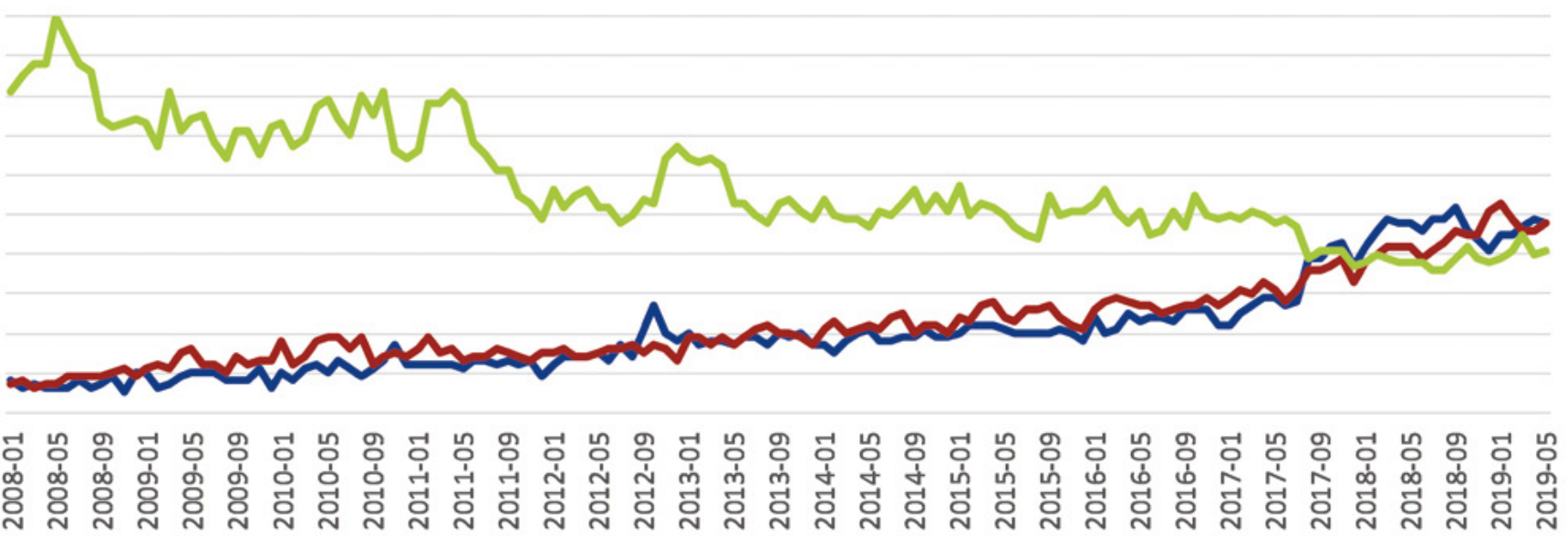

Sciatica Low back pain $\quad$ Multiple sclerosis 


\section{PubMed findings}

2018 PubMed statistics show that scientific interest in sciatica is low. Search query sciatica returned 140 articles. The combined search terms leg pain, lumbar and 2018 return 406 results. The results are slightly higher for low back pain (2,218 publications). This might suggest that scientists are more concerned of the problem of low back pain than sciatica, however, the varying definitions of sciatica in literature do not allow a reliable comparison of the real burden of sciatica and low back pain [9]. Nevertheless, both conditions garner a lower scientific interest when compared to another neurological condition such as multiple sclerosis $(4,817$ articles) as seen on Figure 1. Multiple sclerosis faced a $29 \%$ decrease of in-web searches, a $54 \%$ decrease on YouTube and a $20 \%$ decrease on Google Images between 2008 and 2019. Notably, multiple sclerosis has a far lower prevalence than sciatica or back pain.

\section{Discussion}

We found that there is an overwhelming increase in public interest on sciatica and low back pain. This finding is novel as we could not find any published study describing this trend. Perhaps this is due to the fact that online tools such as Massviews have only recently been available to highlight these trends.

Although effective medical, surgical and alternative treatment methods are available for sciatica, it is important for physicians to be aware of this pervasive condition as there is still a lack of evidence concerning the optimal treatment of lumbar disc-induced sciatica [10]. From an economic standpoint, presenteeism due to sciatica may deteriorate productivity in the workplace and undermine a country's economy [11].

There is miscommunication between patients and surgeons in regards to sciatica treatment [12]. One study found that patients felt that they were not given enough information by their surgeon regarding lumbar decompressive surgery to treat sciatica. However, the surgeons considered that they had expressed sufficient information. Due to the perceived knowledge gap, patients sought medical information from family, friends and the internet [12]. Notably, the topic "disc herniation" (a common cause of sciatica) yields low quality videos on YouTube [13-14]. Moreover, several studies show that the online information about neurological and neurosurgical diseases is too difficult for patients to understand [15-17]. Various treatments for sciatica have been recommended including bed rest, physical therapy, spinal manipulation and lumbar discectomy [18-20]. Given the broad spectrum of treatment options and the current online interest in sciatica, it would be beneficial if the scientific community provided reliable and reader-friendly information for the public.

\section{Limitations of the analysis}

Google accounted for $92 \%$ of the search engine market share worldwide from 2009 to 2018 . Thus about $8 \%$ of the searches conducted on other search engines (i.e. DuckDuckGo, Bing, Yahoo!, Baidu, Yandex RU and Sogu) were not analyzed with Google Trends [21].

The definition of sciatica may differ among information-seekers. Even among physicians the exact definition of sciatica differs. Some researchers define sciatica as leg pain that extends from the back yet others restrict it to pain from specifically the lumbar nerve root [9].

The exact location and demographics of the increased online interest in sciatica could not be determined as it was not available from the platforms analyzed. Future studies may assess the age, gender, location or education level of information-seekers to pinpoint in exactly which populations saw an increase in sciatica.

\section{Conclusion}

Our study highlights that modern online tools may offer concerning public health information-seeking behavior in neurology and neurosurgery. Objective online metrics used in our paper have revealed that sciatica and back pain garner relatively high online interest. We suggest that hospital staff clearly highlight the causes, pathophysiology, diagnosis and management of sciatica in an easy to understand way via in-hospital leaflets, online materials and public relations work. We encourage physicians to be mindful of the high public interest in sciatica to mediate patient expectations regarding their prognosis and sciatica treatment options.

\section{Conflicts of interest: none reported}

Funding: No grant from public, commercial, or not-for-profit sector agencies were obtained for this research project.

Ethical permission: Ethical approval was not necessary for the preparation of this article. 


\section{References}

1. Aitken M, Altmann T, Rosen D. Engaging patients through social media. IMS Inst Healthc informatics, Tech Rep. 2014;

2. Laurent MR, Vickers TJ. Seeking Health Information Online: Does Wikipedia Matter? J Am Med Informatics Assoc [Internet]. 2009 Jul 1;16(4):471-9. Available from: https://academic.oup.com/iamia/article-lookup/doi/10.1197/jamia.M3059

3. Kinney MO, Brigo F. What can Google Trends and Wikipedia-Pageview analysis tell us about the landscape of epilepsy surgery over time? Epilepsy Behav [Internet]. 2020 Feb;103:106533. Available from: https://linkinghub.elsevier.com/ retrieve/pii/S1525505019308650

4. Brigo F, Lattanzi S, Bragazzi N, Nardone R, Moccia M, Lavorgna L. Why do people search Wikipedia for information on multiple sclerosis? Mult Scler Relat Disord [Internet]. 2018 Feb;20:210-4. Available from: https://linkinghub.elsevier. com/retrieve/pii/S2211034818300476

5. Brigo F, Igwe SC, Nardone R, Lochner P, Tezzon F, Otte WM. Wikipedia and neurological disorders. J Clin Neurosci [Internet]. 2015 Jul;22(7):1170-2. Available from: https://linkinghub.elsevier.com/retrieve/pii/S0967586815000995

6. Stafford MA, Peng P, Hill DA. Sciatica: a review of history, epidemiology, pathogenesis, and the role of epidural steroid injection in management. $\mathrm{Br} J$ Anaesth [Internet]. 2007 Oct;99(4):461-73. Available from: https://linkinghub.elsevier. com/retrieve/pii/S0007091217354612

7. Google Inc. Google Trends [Internet]. [cited 2020 Apr 14]. Available from: https://trends.google.com/trends/?geo=UK

8. MusikAnimal, Kaldari, Forns MR. Massviews Analysis [Internet]. [cited 2020 Apr 14]. Available from: https://tools.wmflabs.org/massviews/

9. Lewis R, Williams N, Matar H, Din N, Fitzsimmons D, Phillips C, et al. The clinical effectiveness and cost-effectiveness of management strategies for sciatica: systematic review and economic model. Health Technol Assess (Rockv) [Internet]. 2011 Nov;15(39). Available from: https://www.journalslibrary.nihr.ac.uk/hta/hta15390/

10. Peul WC, van Houwelingen HC, van der Hout WB, Brand R, Eekhof JA, Tans JT, et al. Prolonged conservative treatment or 'early' surgery in sciatica caused by a lumbar disc herniation: rationale and design of a randomized trial [ISRCT 26872154]. BMC Musculoskelet Disord [Internet]. 2005 Dec 11;6(1):8. Available from: http://www.ncbi.n/m.nih.gov/pubmed/15707491

11. Low Back Pain and Sciatica in Over 16s: Assessment and Management [Internet]. National Institute for Health and Care Excellence (UK); 2016. Available from: https://www.ncbi.nlm.nih.gov/pubmed/27929617

12. Rehman Y, Syed M, Wiercioch W, Rehman N, Drew B, Cenic A, et al. Discrepancies Between Patient and Surgeon Expectations of Surgery for Sciatica. Spine (Phila Pa 1976) [Internet]. 2019 May;44(10):740-6. Available from: http://journals. Iww.com/00007632-201905150-00013

13. Gokcen HB, Gumussuyu G. A Quality Analysis of Disc Herniation Videos on YouTube. World Neurosurg [Internet]. 2019 Apr;124:e799-804. Available from: https://linkinghub.elsevier.com/retrieve/pii/S1878875019302463

14. Szmuda T, Ali S, Słoniewski P. Letter to the Editor Regarding "A Quality Analysis of Disk Herniation Videos on YouTube". World Neurosurg [Internet]. 2019 Oct 1 [cited 2020 Mar 24];130:570-2. Available from: https://www.sciencedirect.com/ science/article/pii/S1878875019314482?via\%3Dihub

15. Schmitt PJ, Prestigiacomo CJ. Readability of neurosurgery-related patient education materials provided by the American Association of Neurological Surgeons and the National Library of Medicine and National Institutes of Health. World Neurosurg [Internet]. 2013;80(5):e33-9. Available from: https://doi.org/10.1016/j.wneu.2011.09.007

16. Brigo F, Otte WM, Igwe SC, Tezzon F, Nardone R. Clearly written, easily comprehended? The readability of websites providing information on epilepsy. Epilepsy Behav [Internet]. 2015 Mar;44:35-9. Available from: https://linkinghub.elsevier. com/retrieve/pii/S1525505014006994

17. Modiri O, Guha D, Alotaibi NM, Ibrahim GM, Lipsman N, Fallah A. Readability and quality of wikipedia pages on neurosurgical topics. Clin Neurol Neurosurg [Internet]. 2018 Mar;166:66-70. Available from: https://linkinghub.elsevier.com/ retrieve/pii/S0303846718300271

18. Vroomen PCAJ, de Krom MCTFM, Slofstra PD, Knottnerus JA. Conservative Treatment of Sciatica: A Systematic Review. J Spinal Disord [Internet]. 2000 Dec;13(6):463-9. Available from: http://content.wkhealth.com/linkback/openurl?sid=WKPTLP:landingpage \&an=00002517-200012000-00001

19. Hagen K, Hilde G, Jamtvedt G, Winnem M. Bed rest for acute low-back pain and sciatica. In: Cochrane Database of Systematic Reviews [Internet]. Chichester, UK: John Wiley \& Sons, Ltd; 2000. p. CD001254. Available from: http://www.ncbi. nlm.nih.gov/pubmed/10796429

20. Gibson JA, Waddell G. Surgical interventions for lumbar disc prolapse. In: Gibson JA, editor. Cochrane Database of Systematic Reviews [Internet]. Chichester, UK: John Wiley \& Sons, Ltd; 2007. p. CD001350.

21. Search Engine Market Share Worldwide [Internet]. StatCounter Global Stats. [cited 2020 Apr 14]. Available from: https:// gs.statcounter.com/search-engine-market-share 